\title{
Adaptive Control And Multiple Parameters Identification With Single Differential Equation
}

\author{
Yuqiang Jin ${ }^{1, \mathrm{a}}$, Junwei Lei ${ }^{2, \mathrm{~b}}$, Zexue $\mathrm{Li}^{2}$ \\ ${ }^{1}$ Department of Training, Naval aeronautical and astronautical University, Yantai, China \\ ${ }^{2}$ Department of control engineering, Naval aeronautical and astronautical University, Yantai, China \\ ajinyuqiang1024@126.com, 'Pleijunwei@126.com
}

Keywords: adaptive control, parameter identification, one order system

\begin{abstract}
How to identify multiple parameters quickly and accurately is a key or problem of control system optimization. Based on Terminal attractor and Sigmoid function, an adaptive control law is designed, and the result of parameter identification is analyzed. In the end, a conclusion can be made that theoretical analysis is correct and parameter identification method is effective by numerical simulation.
\end{abstract}

\section{Introduction}

Parameter identification is a complex problem that attracted many researchers in recent years ${ }^{[1-4]}$. But it is still a complex problem especially for high order systems with uncertainty ${ }^{[5-9]}$. Sometimes it is even more difficult to identify the unknown parameter than to design a controller to make the system stable. So in this paper the parameter identification problem for first order system with one differential equation is discussed with terminal control method ${ }^{[10-11]}$. Terminal control has robustness and is not sensitive to parameter variation. Sigmoid function is one of the most transfer function in artificial neural networks, it is used in artificial neural networks[1-3] at the earliest, it has continuity, smooth, differentiability, boundedness. In this paper, Terminal attractor and Sigmoid function will be used in adaptive control to solve the problem of multiple parameter identification.

\section{Problem Description}

One order system can be written as:

$$
\dot{x}=\sum_{i=1}^{m} a_{i} g_{i}(x)+u
$$

where $a$ is unknown constant parameter, the goal is designing a controller such that the system state $x$ can trace the expected value $x^{d}$.

\section{Design Adaptive Identification Controller} then

An ordinary adaptive control method is used as follows, define a error variable as $z_{1}=x_{1}-x_{1}^{d}$,

$$
\dot{z}_{1}=\dot{x}_{1}-\dot{x}_{1}^{d}=\sum_{i=1}^{m} a_{i} g_{i}(x)+u
$$

Design state feedback control law as:

$$
u=-\sum_{i=1}^{m} \hat{a}_{i} g_{i}(x)-\sum_{i=1}^{n} k_{i} f_{i}\left(z_{1}\right)
$$

where $n=5$,

$$
f_{1}\left(z_{1}\right)=z_{1}, \quad f_{2}\left(z_{1}\right)=z_{1}^{3}, \quad f_{3}\left(z_{1}\right)=z_{1}^{1 / 3}
$$




$$
\begin{array}{r}
f_{4}\left(z_{1}\right)=\frac{z_{1}}{\left|z_{1}\right|+\varepsilon}, \quad \varepsilon=0.2, \\
f_{5}\left(z_{1}\right)=\frac{1-e^{-\tau z_{1}}}{1+e^{-\tau z_{1}}}, \quad \tau=0.5
\end{array}
$$

where $f_{3}\left(z_{1}\right)$ is Terminal attractor, and $f_{5}\left(z_{1}\right)$ is Sigmoid function, $f_{4}\left(z_{1}\right)$ and $f_{5}\left(z_{1}\right)$ both have boundedness, Obviously, $f_{i}\left(z_{1}\right)$ meet $z_{1} f_{i}\left(z_{1}\right) \geq 0$, then

$$
\dot{z}_{1}=\sum_{i=1}^{m} \tilde{a}_{i} g_{i}(x)-\sum_{i=1}^{n} k_{i} f_{i}\left(z_{1}\right)
$$

where the error variable $\tilde{a}$ can be defined as:

$$
\tilde{a}_{i}=a_{i}-\hat{a}_{i},
$$

design regulating law:

$$
\dot{\hat{a}}_{i}=\Gamma_{i} z_{1} g_{i}(x)
$$

where $\hat{a}$ is unknown estimated parameter value, choose initial value $\hat{a}(0)=0$, then

$$
\dot{\tilde{a}}_{i}=-\dot{\hat{a}}_{i}
$$

choose Lyapunov function:

$$
V=\frac{1}{2} z_{1}^{2}+\sum_{i=1}^{m} \frac{1}{2 \Gamma_{i}} \tilde{a}_{i}^{2}
$$

then

$$
\dot{V}=z_{1} \dot{z}_{1}+\sum_{i=1}^{m} \frac{1}{\Gamma_{i}} \tilde{a}_{i} \dot{\tilde{a}}_{i}
$$

then

$$
\dot{V}=\sum_{i=1}^{m} z_{1} \tilde{a}_{i} g_{i}(x)-\sum_{i=1}^{n} k_{i} z_{1} f_{i}\left(z_{1}\right)-\sum_{i=1}^{m} \frac{1}{\Gamma_{i}} \tilde{a}_{i} \Gamma_{i} z_{1} g_{i}(x)=-\sum_{i=1}^{n} k_{i} z_{1} f_{i}\left(z_{1}\right) \leq 0
$$

So $Z_{1} \rightarrow 0$.

\section{Parameter Identification Result Analysis}

When $z_{1} \rightarrow 0$, where $u=-\sum_{i=1}^{m} \hat{a}_{i} g_{i}(x)$, then

$$
\dot{z}_{1}=\sum_{i=1}^{m} \tilde{a}_{i} g_{i}(x)
$$

So when $z_{1} \rightarrow 0$, where $\dot{z}_{1} \rightarrow 0, \quad \dot{z}_{1}=\sum_{i=1}^{m} \tilde{a}_{i} g_{i}(x)=0$. If $g_{i}(x)$ are uncorrelated, then $\tilde{a}_{i} \rightarrow 0$ 。

\section{Numerical Simulation}

Choose $n=2, a_{1}=5, a_{2}=-5, g_{1}(x)=x, g_{2}(x)=\sin (x), x_{1}^{d}=2$, program can be written as follows:

clc;clear; $1=5 ; \mathrm{a} 2=-5 ; \mathrm{x} 1 \mathrm{~d}=2 ; \mathrm{a} 1 \mathrm{~g}=0 ; \mathrm{a} 2 \mathrm{~g}=0 ; \mathrm{x}=0 ; \mathrm{u}=0 ; \mathrm{tf}=15 ; \mathrm{dt}=0.001$;

for $\mathrm{i}=1: \mathrm{tf} / \mathrm{dt}$

$\mathrm{t}=\mathrm{i} * \mathrm{dt} ; \mathrm{g} 1 \mathrm{x}=\mathrm{x} ; \mathrm{g} 2 \mathrm{x}=\mathrm{x} \wedge 3$

$\mathrm{dx}=\mathrm{a} 1 * \mathrm{~g} 1 \mathrm{x}+\mathrm{a} 2 * \mathrm{~g} 2 \mathrm{x}+\mathrm{u} ; \quad \mathrm{x}=\mathrm{x}+\mathrm{dx} * \mathrm{dt}$;

$\mathrm{k} 1=5 ; \mathrm{k} 2=5 ; \mathrm{k} 3=1 ; \mathrm{k} 4=1 ; \mathrm{k} 5=1 ;$ esten $=0.2 ; \mathrm{tao}=0.5$; 
$\mathrm{k} 1=5 ; \mathrm{k} 2=5 ; \mathrm{k} 3=5 ; \mathrm{k} 4=5 ; \mathrm{k} 5=5$;

$\mathrm{z} 1=\mathrm{x}-\mathrm{x} 1 \mathrm{~d} ; \mathrm{f} 1=\mathrm{z} 1 ; \mathrm{f} 2=\mathrm{z} 1 \wedge 3 ; \mathrm{f} 3=\mathrm{z} 1 \wedge(1 / 3) ; \mathrm{f} 4=\mathrm{z} 1 /(\operatorname{abs}(\mathrm{z} 1)+\mathrm{esten}) ;$

$\mathrm{f} 5=\left(1-\exp \left(-\mathrm{tao}^{*} \mathrm{z} 1\right)\right) /\left(1+\exp \left(-\mathrm{tao}^{*} \mathrm{z} 1\right)\right)$;

ta1=5;ta2=5;da1g=ta1*g1x*z1;da2g=ta2*g2x*z1;

$\mathrm{a} 1 \mathrm{~g}=\mathrm{a} 1 \mathrm{~g}+\mathrm{da} 1 \mathrm{~g} * \mathrm{dt} ; \mathrm{a} 2 \mathrm{~g}=\mathrm{a} 2 \mathrm{~g}+\mathrm{da} 2 \mathrm{~g} * \mathrm{dt} ;$

$\mathrm{u}=-\mathrm{a} 1 \mathrm{~g} * \mathrm{~g} 1 \mathrm{x}-\mathrm{a} 2 \mathrm{~g} * \mathrm{~g} 2 \mathrm{x}-\mathrm{k} 1 * \mathrm{f} 1-\mathrm{k} 2 * \mathrm{f} 2-\mathrm{k} 3 * \mathrm{f} 3-\mathrm{k} 4 * \mathrm{f} 4-\mathrm{k} 5 * \mathrm{f} 5$;

end

tp(i) $=t ; x p(i)=x ; a 1 g p(i)=a 1 g ; a 2 g p(i)=a 2 g$;

figure(1);plot(tp,xp,'k');xlabel('t/s');ylabel('state x');

figure(2);plot(tp,a1gp,'k');xlabel('t/s');ylabel('state a1g');

figure(3);plot(tp,a2gp,'k');xlabel('t/s');ylabel('state a2g');

And the simulation results are as follows:

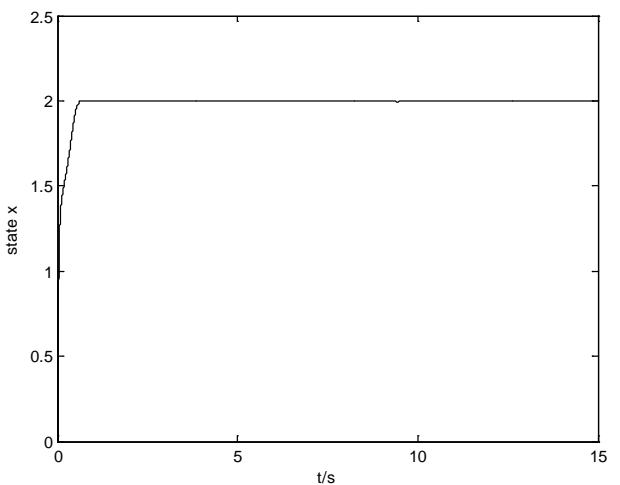

Fig. 1 state $\mathrm{x}$

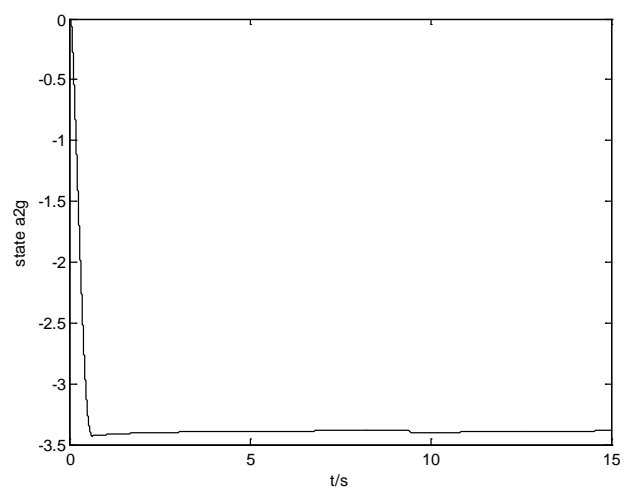

Fig.3 state a2

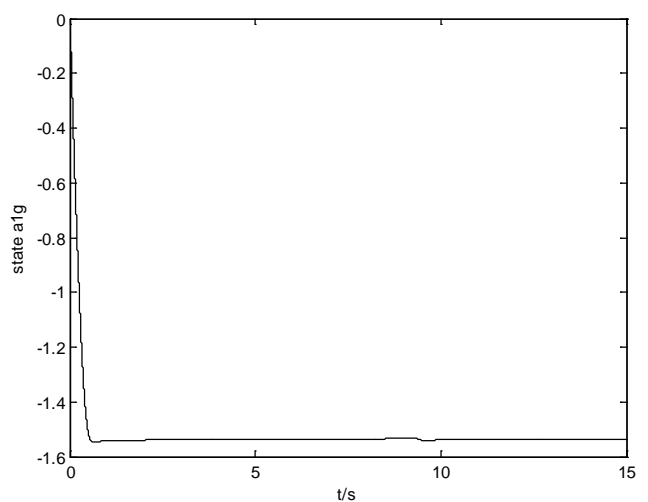

fig.2 state a1

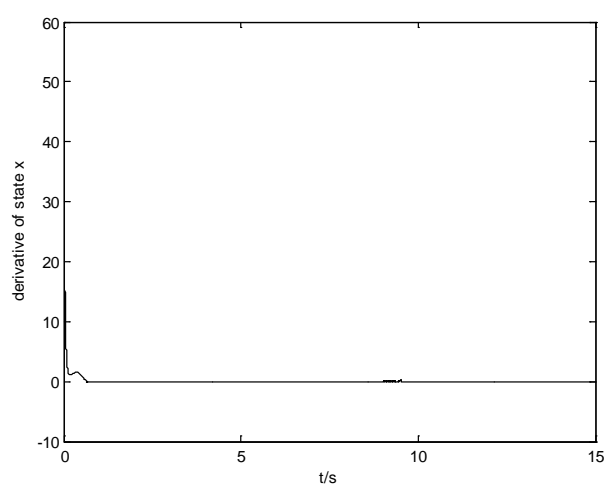

fig.4 state $\mathrm{x}$

From the simulation results, we can know that system state can track expected state, but parameter identification can not be achieved. The main reason is that estimated parameter error can balance at optional position when final state $x$ is constant.

Consider input is periodic signal, as $u=\sin (t)$, program can be written as follows: clc;clear;a1=5;a2=-5; $1 \mathrm{~d}=2 ; \mathrm{a} 1 \mathrm{~g}=0 ; \mathrm{a} 2 \mathrm{~g}=0 ; \mathrm{x}=0 ; \mathrm{u}=0 ; \mathrm{tf}=150 ; \mathrm{dt}=0.001$;

for $\mathrm{i}=1: \mathrm{tf} / \mathrm{dt}$

$\mathrm{t}=\mathrm{i}^{*} \mathrm{dt} ; \mathrm{g} 1 \mathrm{x}=\mathrm{x} ; \mathrm{g} 2 \mathrm{x}=\mathrm{x}^{\wedge} 3 ; \quad \mathrm{x} 1 \mathrm{~d}=\sin (\mathrm{t}) ;$

$\mathrm{dx}=\mathrm{a} 1 * \mathrm{~g} 1 \mathrm{x}+\mathrm{a} 2 * \mathrm{~g} 2 \mathrm{x}+\mathrm{u} ; \quad \mathrm{x}=\mathrm{x}+\mathrm{dx} * \mathrm{dt}$;

$\mathrm{k} 1=5 ; \mathrm{k} 2=5 ; \mathrm{k} 3=1 ; \mathrm{k} 4=1 ; \mathrm{k} 5=1 ; \mathrm{esten}=0.2 ; \mathrm{tao}=0.5$;

$\mathrm{k} 1=5 ; \mathrm{k} 2=5 ; \mathrm{k} 3=5 ; \mathrm{k} 4=5 ; \mathrm{k} 5=5$;

$\mathrm{z} 1=\mathrm{x}-\mathrm{x} 1 \mathrm{~d} ; \mathrm{f} 1=\mathrm{z} 1 ; \mathrm{f} 2=\mathrm{z} 1 \wedge 3 ; \mathrm{f} 3=\mathrm{z} 1 \wedge(1 / 3) ; \mathrm{f} 4=\mathrm{z} 1 /(\mathrm{abs}(\mathrm{z} 1)+\mathrm{esten}) ;$

f5=(1-exp $\left(-\right.$ tao*z1 $\left.^{2}\right) /\left(1+\exp \left(-\right.\right.$ tao*z1 $\left.^{2}\right)$;

ta1=5; ta2=5; da1g=ta1*g1x*z1; da2g=ta2*g2x*z1; a1g=a1g+da1g*dt;a2g=a2g+da2g*dt;

u=-a1g*g1x-a2g*g2x-k1*f1-k2*f2-k3*f3-k4*f4-k5*f5; 
end

$\operatorname{tp}(i)=t ; x p(i)=x ; a 1 g p(i)=a 1 g ; a 2 g p(i)=a 2 g ; \quad d x p(i)=d x ; x 1 d p(i)=x 1 d ; z 1 p(i)=z 1 ;$

figure(1);plot(tp,xp,'k');xlabel('t/s');ylabel('state x');

figure(2);plot(tp,a1gp,'k');xlabel('t/s');ylabel('state a1g');

figure(3);plot(tp,a2gp,'k');xlabel('t/s');ylabel('state a2g');

figure(4);plot(tp,dxp,'k');xlabel('t/s');ylabel('derivative of state x');

figure(5);plot(tp,z1p,'k');xlabel('t/s');ylabel('z1');

And the simulation results are as follows:

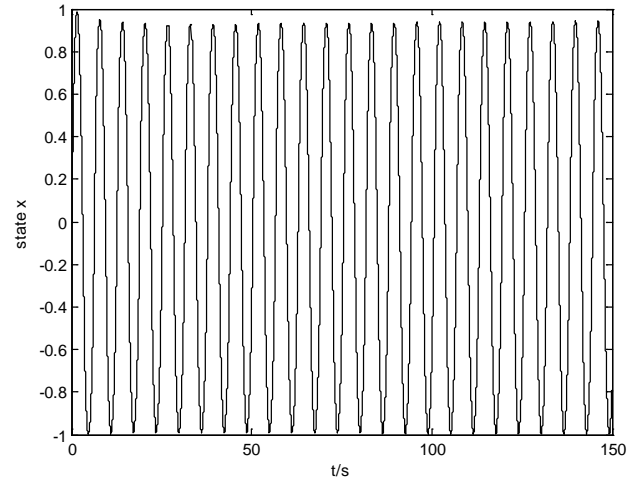

Fig. 5 state $\mathrm{x}$

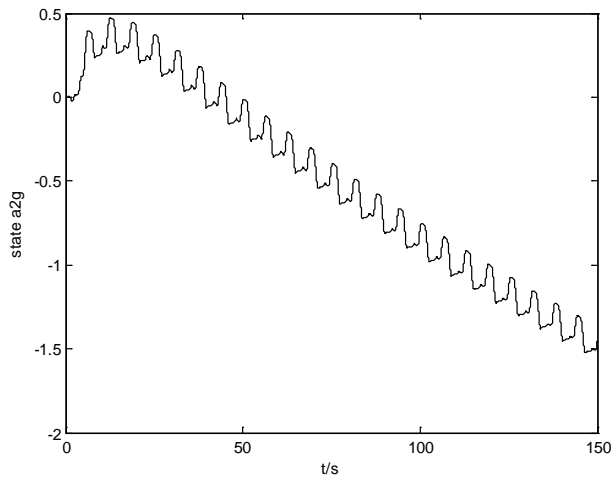

Fig.7 state a2

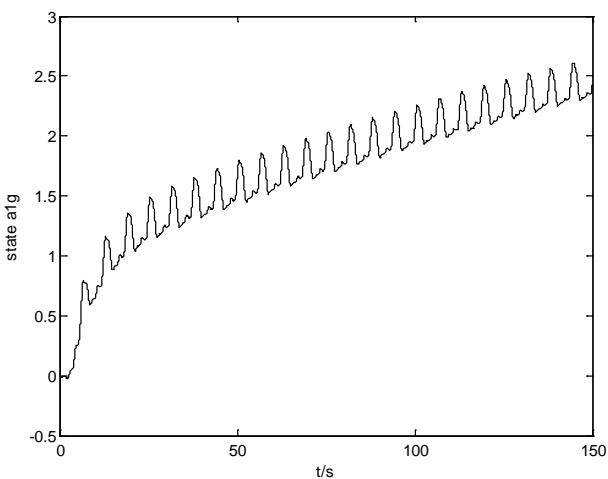

fig.6 state a1

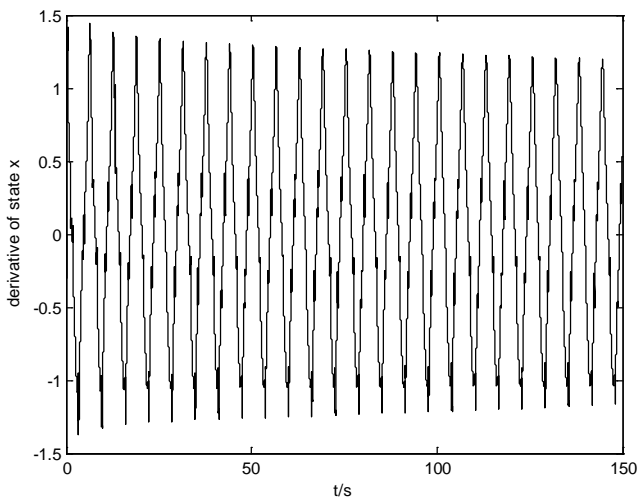

fig.8 state $\mathrm{x}$

From the simulation results, we can see that the trend of unknown parameter identification is correct. According to the simulation results, when expected signal is a constant, system error can converge to zero quickly, but the result of parameter identification is not ideal, the main reason is that multiple parameter identification offset.

\section{Conclusion}

By analyzing theory and the simulation results, we can make a conclusion that the method of combining Terminal and Sigmoid function in this paper is effective to parameter identification. When the control gain is matched with identification gain, unknown parameter identification can success easily. But multiple parameter identification offset, the result is not ideal. The input signal should be considered.

\section{Reference}

[1] Carrasco R C, Forcada M L. Simple strategies to encode tree automata in sigmoid recursive neural networks[J]. IEEE Transactions on Knowledge and Data Engineering 2001, 13(2): 148-156.

[2] Basterretxea K, Tarela J M. Del Campo I. Implementation issue of sigmoid function and its derivative for VLSI digital neural networks[J]. Electronics Letters, 2002, 38(1). 
[3]Basterretxea K, Tarela J M. Del Campo I. Approximation of sigmoid function and derivative for hardware implementation of artificial neurons[j]. IEEE Proceedings online, 2004, 151(1).

[4] Lorenz EN. Deterministic non-periods flows. J Atmos Sci 1963;20:130-41.

[5] Chen GR, Ueta T. Yet another chaotic attractor. Int J Bifurc Chaos 1999;9:1465-6.

[6] Lv JH, Chen G.R A new chaotic attractor coined. Int J Bifurc Chaos 2002;12(3):659-61.

[7] Van-ec-ek A, C-elikovesky S. Control systems: from linear analysis to synthesis of chaos. London: Prentice-Hall; 1996.

[8] Wu X, Lu J. Parameter identification and backstepping control of uncertain Lv system. Chaos, Solitons \& Fractals 2003;18:721-9.

[9] Yu Y, Zhang S. Adaptive backstepping control of the uncertain Lvu system. Chin Phys 2002;11:1249-305.

[10] Wang C, Ge S. Adaptive backstepping control of uncertain Lorenz system. Int J Bifurc Chaos 2001;11:1115-9.

[11]E.M.Elabbasy, H.N.Agiza, M.M. El-Dessoky, Adaptive synchronization of a hyperchaotic system with uncertain parameter, Chaos, Solitons and Fractals 30(2006) 1133-1142. 